\title{
List of reviewers
}

We would like to express our gratitude to the following people who provided assessments of at least one manuscript submitted to Archives of Polish Fisheries in 2016

R. M. Adamek (Germany)

E.A. Aragon-Noriega (Mexico)

A.M. Banos (Spain)

R. Bernaś (Poland)

M. Biernaczyk (Poland)

G. Biswas (India)

E. Bogacka-Kapusta (Poland)

C.J. Bugle (USA)

B.I. Cejko (Poland)

I. Chater (Tunisia)

M. Corales (Bangladesh)

R. Czerniawski (Poland)

A. Didenko (Ukraine)

Z. Doðu (Turkey)

S. Eriksonn (Sweden)

K.M. Filho (Brazil)

D. Giannetto (Turkey)

S.S. Giri (Republic of Korea)

K. Goryczko (Poland)

S.H. Hoseinifar (Iran)

M.H. Johnson (United Kingdom)

M. Kamaszewski (Poland)

R. Kamiński (Poland)

P. Kaur (India)

L. Kirczuk (Poland)

J. Kristan (Czech Republic)

J. Król (Poland)

M. Kuciński (Poland)

S. Kumar (India)
Y. Kutsokon (Ukraine)

Y-Ch. Lee (Taiwan)

$\mathrm{X}$. Lin (China)

J. Łuczyńska (Poland)

S.K. Maitra (India)

A. Martyniak (Poland)

N. Matvienko (Ukraine)

D.K. Moutopoulos (Greece)

J. Naslund (Sweden)

N. Nowak (Poland)

A.S. Ochrem (Poland)

P.M. Oglęcki (Poland)

J.M. Oresti (Italy)

J. Rechulicz (Poland)

A.F. Ruttner (USA)

A. Rymuszka (Poland)

M.Z. Saad (Malaysia)

B. Schultz (USA)

M.R. Schwemm (USA)

M.A.M. Siddique (Czech Republic)

K. Skieresz-Szewczyk (Poland)

S. Sobhanardakani (Iran)

F. Stein (Germany)

T.R. Ulvick (USA)

P. Vila (Spain)

M. Witeska (Poland)

V. Yancheva (Bulgaria)

S. Yilmaz (Turkey)

B. Zdanowski (Poland)

D. Żarski (Poland) 